\title{
Determination of Atherosclerosis markers changes after HIIT and ginger consumption in response to acute exercise in overweight women
}

\author{
Mohammad Esmaeil Afzalpour ${ }^{1}$, Shila Nayebifar ${ }^{*}$, Tooba Kazemi ${ }^{2}$, Seyed-Hosein Abtahi-Eivary ${ }^{3}$, Mehdi Mogharnasi ${ }^{1}$ \\ ${ }^{1}$ Department of Exercise Physiology, Faculty of Physical Education and Sport Sciences, University of Birjand, Birjand, Islamic Republic of Iran. \\ ${ }^{2}$ Atherosclerosis and Coronary Artery Research Centre, Department of Cardiology, Birjand University of Medical Sciences, Birjand, Islamic Republic of \\ Iran. ${ }^{3}$ Department of Biochemistry, Faculty of Medicine, Gonabad University of Medical Sciences, Gonabad, Islamic Republic of Iran.
}

\begin{tabular}{|c|c|}
\hline ARTICLE INFO & ABSTRACT \\
\hline Article history: & \multirow{11}{*}{$\begin{array}{l}\text { The purpose of this study was to determine the effects of ten weeks of high intensity interval training (HIIT) } \\
\text { with ginger supplementation on the systemic inflammatory response to acute HIIT exercise. Twenty four } \\
\text { sedentary overweight women randomly assigned into three groups' including HIIT + ginger ( } \mathrm{n}=8 \text { ), HIIT }+ \\
\text { placebo }(\mathrm{n}=8) \text { and ginger }(\mathrm{n}=8 \text { ). Sixteen women performed ten weeks of HIIT while the supplement consuming } \\
\text { groups daily took } 3 \text { gm. of ginger pills and the HIIT }+ \text { placebo group took placebo. Serum samples were } \\
\text { collected at rest and immediately post-acute exercise, before and after the training. Two-way repeated measures } \\
\text { ANOVA, paired t test, one-way ANOVA and post hoc Bonferroni were used to analyze data. Results indicated } \\
\text { that three groups similarly affect the Monocyte Chemo tactic protein-1 (MCP-1) and Interlukine-10 (IL-10) } \\
\text { changes in response to acute exercise (p>0.05) whereas significant increase in intercellular adhesion molecule-1 } \\
\text { (ICAM-1) in HIIT + placebo group in comparison with HIIT + ginger group (p=0.02) in second acute exercise } \\
\text { was observed. The combination of HIT and ginger consumption may induce a modest inflammatory attenuation } \\
\text { of atherosclerosis factors in response to acute exercise and improvement in } \mathrm{Vo}_{2} \text { max and PBF results rather than } \\
\text { ginger and training alone, in young sedentary overweight women. }\end{array}$} \\
\hline Received on: $24 / 05 / 2016$ & \\
\hline Revised on: 13/06/2016 & \\
\hline Accepted on: 06/07/2016 & \\
\hline Available online: $28 / 07 / 2016$ & \\
\hline & \\
\hline Training, Inflammatory & \\
\hline Cytokines, Exercise, Ginger. & \\
\hline & \\
\hline & \\
\hline & \\
\hline
\end{tabular}

\section{INTRODUCTION}

Scientific documents agree the hypothesis that functions of inflammation in the initiation, progression and plaque rupture of the acute coronary disease, atherosclerosis (Libby et al., 2002). In this phenomena, a variety of inflammatory biomarkers have been identified, some of them act as primary markers in the first stages of progression. Adhesion and attraction of monocytes to intima is the role of inter cellular adhesion molecule-1(ICAM-1) a vascular cell adhesion molecule which can independently predict cardiovascular diseases (CVD)(Ridker et al., 1998) and monocyte chemotactic protein-1

\footnotetext{
* Corresponding Author

Shila Nayebifar, Department of Exercise Physiology, Faculty of Physical Education and Sport Sciences, University of Birjand, Birjand, Islamic Republic of Iran.Email: shila_neyebi@yahoo.com
}

(MCP-1) an inflammatory chemokine, respectively (Ribeiro et al., 2012). It seems that some of athero-protective agents are present in the lesion to attenuate the inflammatory cascade. Interlukine-10 (IL-10) is one of the key anti-inflammatory cytokines, a polytropic interleukin, which acts in suppression of many inflammatory factors expression such as ICAM-1 and MCP-1 through inhibition

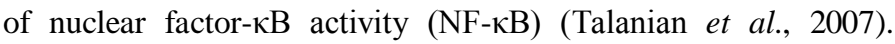
However, available evidence suggests that concentration of ICAM1, MCP-1and IL-10 can be modulating by pro inflammatory conditions (Vrousgos, 2015), exercise training (Ribeiro et al., 2012) or after coupling with some of natural ingredients of herbs as seen by ginger (Wang et al., 2013).

Among non-pharmacological strategies in improving cardiovascular diseases, exercise training and herbal medicine has been considered effective. Ginger a well-known spice plant has been used to treat cardiovascular disorders (atherosclerosis) (Nicoll and Henein, 2009). 
It has been earlier reported that the anti-inflammatory action of the main component of ginger (gingerols or shogaol) is by inhibition of prostaglandin and leukotriene biosynthesis through suppression of 5-lipoxygenase or prostaglandin synthesis. Additionally, they can also inhibit synthesis of pro-inflammatory cytokines such as IL-1, TNF- $\alpha$, and IL-8 (Verma et al., 2004). Pan et al. (2008) showed that in macrophages, 6-shogaol can downregulate inflammatory inducible nitric oxide synthesis (iNOS) and cycloxygenasis-2 (COX-2) gene expression (Pan et al., 2008). It has been earlier reported that ginger induced positive effects on ICAM-1 and MCP-1 production in vitro (Wang et al., 2013) and some of inflammatory markers in vivo (Fatehi et al., 2005). Exercise training, the other therapeutic approach, accounts for modulating inflammation (Sallam and Laher, 2015). In this context, chronic treadmill running (Simpson et al., 2006), low to moderate aerobic protocols (Abd El-Kader et al., 2013) could improve the inflammation. In addition, strenuous physical activities are associated with an inflammatory response involving the activation of adhesion molecules (Nielsen and Lyberg, 2004) and chemokines (Smith et al., 2007). Currently, the high intensity interval trainings (HIIT) were outspread, because of, lesser training volume, making it a time-efficient approach with increased health benefits compared with continuous aerobic exercise training (Fatehi et al., 2005) and it has also been reported as "more enjoyable" than continuous moderate-intensity exercise in healthy young subjects (Bartlett et al., 2014).

Moreover, counteractive effects of acute and chronic exercise especially with high intensity interval protocols concurrently, on mentioned selective atherosclerotic indices have not been investigated before. The aim of the present study was to assess the effects of 10 weeks of HIIT and ginger consumption as two non-pharmacological therapeutic methods, on serum ICAM-1, MCP-1 and IL-10 concentration responses to an acute exercise in overweight women.

\section{MATERIALS AND METHODS}

\section{The study design}

Thirty overweight young women (aged 20-30 years and body mass index $>25 \mathrm{~kg} / \mathrm{m}^{2}$ ) volunteered to participate in this randomized, placebo-controlled study. The Ethics Committee of Birjand University of Medical Sciences (Iran) has approved the study proposal. After explaining the purpose, possible risks and discomforts of study to the participants, and gathering their written informed consent, subjects were randomly assigned to three groups of ten, according to age, body mass index (BMI), maximal oxygen consumption $\left(\mathrm{Vo}_{2} \mathrm{max}\right)$, namely, HIIT + ginger, HIIT + placebo and Ginger. At the end of program, 2 persons in each group didn't complete the procedure so eliminated from study. Before inclusion in the study, an echocardiography examination was conducted for each subject to confirm the cardiac structural and functional health. Exclusion criteria included physical exercise more than 1 time a week, BMI $<25 \mathrm{~kg} / \mathrm{m}^{2}$, smoking, having history of neurological, cardiovascular, respiratory, diabetes, musculoskeletal diseases, use of medication and supplementation with ginger or anti-oxidants and multivitamins. Subjects were weighed while nude, using calibrated precision weighing scales (TCM, China) both before and after the protocol. Percent body fat was determined using Jackson-Pollock 3 Site Skin fold procedure with caliper (Yagami, Japan, EIYOKEN-Type, PAT 376843) (Jackson et al., 1985). $\mathrm{Vo}_{2} \max$ was conducted using the Bruce protocol one week before and after the study design (Wilmore and Costill, 2005).The schematic illustration of the experimental design is shown in Fig 1.

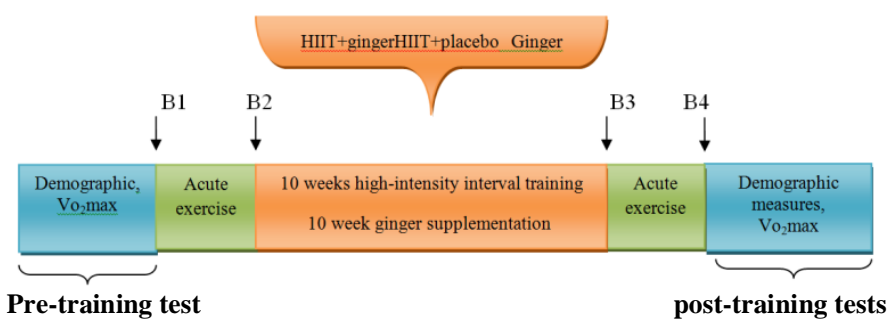

Fig 1: Schematic Illustration of the Experimental Design.B1: blood sampling at pre-exercise, pre-training;B2: blood sampling at post-exercise, pre-training;B3: blood sampling at pre-exercise, post-training; B4: blood sampling at postexercise, post-training.

\section{The supplement intake procedure}

Participants in the HIIT + ginger and Ginger groups received Ginger tablets (namely Vomigan Dineh Iran Co., 3 grams daily), while the HIIT + placebo group received placebo tablets (Gram flour) produced in Dineh Iran Company, for 10 weeks.

\section{The high intensity interval training protocol}

The HIIT protocol was implemented for 10 weeks-3 sessions per week and 30sessions in total. The HIIT was as follow: $30 \mathrm{~s}$ maximal effort sprint within a $20-\mathrm{m}$ distance $(40 \mathrm{~m}$ maximal shuttle run test) (Buchan et al., 2011) (Fig 2) at 8 o'clock in the morning. Participants were instructed to sprint maximally for a period of $30 \mathrm{~s}$, following $30 \mathrm{~s}$ active rest, and repeat this procedure a further three times, which equated to 2 min of maximal effort sprinting interspersed with 2 min recovery. In order to increase the training progression, after each two weeks, one rep was added (from 4 reps in $1^{\text {th }}$ to 8 reps in $10^{\text {th }}$ week) (Buchan et al., 2011). Each training session consisted of 5-10 min warm-up and cooldown. Maximum heart rate (HRmax) (220- age) was used to determine training intensity, which was above $90 \%$ HRmax in all the stages, evaluated conclusively for each subject so all subjects carried a Pollar Telemetry (made in Finland). In contrast, subjects in ginger group performed no training during protocol.

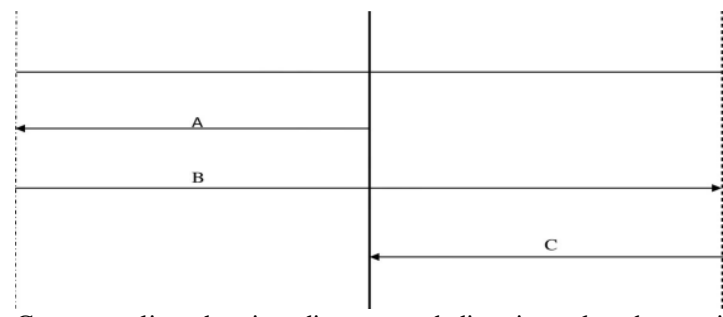

Fig. 2: Course outline showing distance and direction taken by participants, during the $30 \mathrm{~s}$ HIIT protocol. $\mathrm{A}=10 \mathrm{~m}, \mathrm{~B}=20 \mathrm{~m}, \mathrm{C}=10 \mathrm{~m}$. 


\section{The acute exercise protocol}

The acute exercise trial was started with a brief warm-up (cycling at $50 \mathrm{~W}$ for $5 \mathrm{~min}$ ) followed immediately by the acute HIIT protocol $(4 \times 30-s$ all-out cycling at a constant load corresponding to $0.075 \mathrm{~kg} / \mathrm{kg}$ body mass i.e., Wingate tests) separated by 4 min of active rest on an electronically braked ergo meter (Monark, 894E, Sweden) (Little et al., 2011) Heart rate was collected during exercise using telemetry (Pollar, Finland). Changes in plasma volume were calculated as described by Dill and Costill (1974). Then; the indices were corrected for changes in plasma volume.

\section{Biochemical analyzing}

One day before and $72 \mathrm{hrs}$ after the last training session, subjects arrived in the laboratory after 10-12 hrs fasting, Blood samples $(\sim 10 \mathrm{~mL})$ were drawn from a superficial vein in the forearm, using standard vein puncture techniques immediately before and after the acute exercise on ergometer while subjects were in their follicular phase of menstruation (from the first to midpoint of the phase) (Sijie et al., 2012). Samples were then centrifuged at $3000 \mathrm{r} \min -1$ for $15 \mathrm{~min}$ at $4^{\circ} \mathrm{C}$. Following centrifugation, serum was stored at $-80 \mathrm{C}$ for later analysis. Samples were analyzed for serum ICAM-1 (Kazabayo, China), MCP-1(Kazabayo, China) and IL-10 (Diaclone, France) concentration. The intraassay coefficient of variation for variables were as follow: ICAM-1 $<8 \%$, MCP- $1<8 \%$, and IL-10=3.5\%.

\section{The statistical analyzing}

The SPSS software (v.16) was employed for data analysis at a significance level of 0.05. The normality of the study variable was assessed by conducting the Kolmogorov-Smirnov test. Moreover the paired-samples $t$ test was conducted for assessing pretest-posttest within-group variations (Changes in $\mathrm{VO}_{2}$ max and PBF), while changes in the serum ICAM-1, MCP-1 and IL-10 mean were assessed using two-way repeated measures ANOVA, where the within factor was acute exercise (pre-exercise vs. post-exercise) and the between factor was training status (HIIT + ginger vs. HIIT + placebo vs. ginger). For the data that showed a significant interaction effect, Bonferroni post hoc test and one way ANOVA, were used to locate the differences.

\section{RESULTS}

The mean \pm standard deviations of pretest values of the subject's characteristics in three groups are shown in table1 and 2. There were no significant differences in baseline levels of all variables among groups, as determined by 1-way ANOVA test (table 1 and 2).

\section{Alterations in physiological and body composition variables following training}

As shown in table 2, there was significant difference in training induced increase in $\mathrm{Vo}_{2}$ max between groups. The Post Hoc test, Bonferroni, showed significant differences between HIIT + ginger and Ginger $(P=0.000)$, HIIT+ placebo and Ginger groups $(P=0.000)$. On the other hand, paired-t test confirmed significant increase of $\mathrm{Vo}_{2} \mathrm{max}$ in $\mathrm{HIIT}+$ ginger and $\mathrm{HITT}+$ placebo compared to pre-test (table2). In addition, PBF decreased significantly only in HIIT+ Ginger group with significant difference between three groups (table2). The Bonferroni test, showed significant decrease in HIIT + ginger compared to Ginger group $(P=0.01)$.

Table 1: Comparison of the Personal Characteristics of each group at baseline.

\begin{tabular}{|c|c|c|c|c|}
\hline \multirow[b]{2}{*}{ Variable } & \multicolumn{4}{|c|}{ Groups } \\
\hline & $\begin{array}{c}\text { HIIT+ginger } \\
(\text { mean } \pm \text { SD })\end{array}$ & $\begin{array}{c}\text { HIIT+placebo } \\
(\text { mean } \pm \text { SD })\end{array}$ & $\begin{array}{c}\text { Ginger } \\
(\text { mean } \pm \text { SD }) \\
\end{array}$ & $\begin{array}{c}P \text { value } \\
\text { (One-Way Anova) }\end{array}$ \\
\hline Age (year) & $21.87 \pm 3.39$ & $22.37 \pm 3.24$ & $21.62 \pm 1.76$ & 0.87 \\
\hline Height (m) & $1.61 \pm 0.07$ & $1.60 \pm 0.08$ & $1.59 \pm 0.06$ & 0.94 \\
\hline Weight (kg) & $74.19 \pm 11.53$ & $72.24 \pm 6.86$ & $64.91 \pm 3.60$ & 0.07 \\
\hline BMI $\left(\mathrm{kg} / \mathrm{m}^{2}\right)$ & $28.68 \pm 2.60$ & $28.42 \pm 2.40$ & $26.06 \pm 1.68$ & 0.06 \\
\hline $\operatorname{PBF}(\%)$ & $52.89 \pm 4.55$ & $50.50 \pm 10.38$ & $43.61 \pm 5.90$ & 0.11 \\
\hline $\mathrm{Vo}_{2} \max (\mathrm{ml} . \mathrm{kg} \cdot \min )$ & $34.38 \pm 4.89$ & $33.59 \pm 3.16$ & $36.28 \pm 1.29$ & 0.74 \\
\hline
\end{tabular}

Abbreviations: BMI, body mass index; ANOVA, analysis of variance; PBF, percent body fat; $\mathrm{Vo}_{2}$ max, maximum oxygen consumption.

Table 2: The Results Of Between-and Within-Group Comparisons Regarding the Level of Maximal Oxygen Consumption and Percent Body Fat Indices.

\begin{tabular}{|c|c|c|c|c|c|c|}
\hline Variables & Groups & $\begin{array}{l}\text { Pre-training } \\
(\text { mean } \pm \text { SD })\end{array}$ & $\begin{array}{l}\text { Post-training } \\
(\text { mean } \pm \text { SD })\end{array}$ & $\begin{array}{c}\text { PValue (Paired- } \\
\text { t test) }\end{array}$ & $\begin{array}{c}\text { Mean Differences } \\
(\text { mean } \pm \text { SD })\end{array}$ & $\begin{array}{c}P \text { Value } \\
\text { (One-Way ANOVA) }\end{array}$ \\
\hline \multirow{3}{*}{$\begin{array}{c}\mathrm{Vo}_{2} \max \\
\left(\mathrm{ml} \cdot \mathrm{kg}^{-1} \cdot \mathrm{min}^{-1}\right)\end{array}$} & HIIT+ginger & $34.38 \pm 4.89$ & $46.64 \pm 3.08^{a}$ & 0.002 & $-1.22 \mathrm{E} 1 \pm 7.34$ & \multirow{3}{*}{$0.000^{\mathrm{b}}$} \\
\hline & HIIT+placebo & $33.58 \pm 3.16$ & $47.08 \pm 5.30^{\mathrm{a}}$ & 0.000 & $-1.19 \mathrm{E} 1 \pm 4.29$ & \\
\hline & Ginger & $36.28 \pm 1.29$ & $36.07 \pm 1.92$ & 0.12 & $-2.61 \pm 4.27$ & \\
\hline \multirow{3}{*}{ Percent body fat (\%) } & HIIT+ginger & $52.89 \pm 4.55$ & $46.21 \pm 4.50^{\mathrm{a}}$ & 0.04 & $6.67 \pm 4.76$ & \multirow{3}{*}{$0.005^{\mathrm{b}}$} \\
\hline & HIIT+placebo & $50.50 \pm 10.38$ & $41.30 \pm 8.01$ & 0.19 & $4.20 \pm 8.29$ & \\
\hline & Ginger & $43.61 \pm 5.90$ & $46.25 \pm 1.87$ & 0.19 & $-2.36 \pm 5.17$ & \\
\hline
\end{tabular}

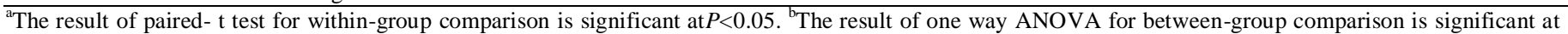
$P<0.05$. 
Table 3: Participants Biochemical Markers in Different Stages of Protocol (Mean \pm SD).

\begin{tabular}{|c|c|c|c|c|c|}
\hline Groups & & ime & $\begin{array}{c}\text { ICAM-1 } \\
(\text { Mean } \pm \text { SD })\end{array}$ & $\begin{array}{c}\text { MCP-1 } \\
(\text { Mean } \pm \text { SD })\end{array}$ & $\begin{array}{c}\text { IL-10 } \\
(\text { Mean } \pm \text { SD })\end{array}$ \\
\hline \multirow{4}{*}{ HIIT + ginger } & \multirow{2}{*}{ Pre-train } & Pre-exercise & $9028.23 \pm 2452.08$ & $103.96 \pm 64.01$ & $3.58 \pm 1.12$ \\
\hline & & Post-exercise & $9781.33 \pm 2525.62$ & $81.54 \pm 47.42$ & $4.44 \pm 1.49$ \\
\hline & \multirow{2}{*}{ Post-train } & Pre-exercise & $7287.87 \pm 1156.45$ & $43.88 \pm 24.56$ & $2.61 \pm 1.63$ \\
\hline & & Post-exercise & $7362.99 \pm 1676.56$ & $53.40 \pm 23.79$ & $2.73 \pm 2.12$ \\
\hline \multirow{4}{*}{ HIIT + placebo } & \multirow{2}{*}{ Pre-train } & Pre-exercise & $7578.42 \pm 540.32$ & $53.79 \pm 17.48$ & $2.12 \pm 0.42$ \\
\hline & & Post-exercise & $9047.91 \pm 1289.03^{a}$ & $48.57 \pm 19.85$ & $4.52 \pm 1.69$ \\
\hline & \multirow{2}{*}{ Post-train } & Pre-exercise & $8785.15 \pm 643.92$ & $29.81 \pm 14.25$ & $3.39 \pm 1.80$ \\
\hline & & Post-exercise & $10603.37 \pm 1131.71^{a}$ & $59.16 \pm 36.23^{\mathrm{a}}$ & $2.78 \pm 1.77^{\mathrm{a}}$ \\
\hline \multirow{4}{*}{ Ginger } & \multirow{2}{*}{ Pre-train } & Pre-exercise & $7018.12 \pm 1531.67$ & $61.96 \pm 30.58$ & $2.12 \pm 0.42$ \\
\hline & & Post-exercise & $8394.59 \pm 430.66^{\mathrm{a}}$ & $43.32 \pm 15.55$ & $2.86 \pm 1.50$ \\
\hline & \multirow{2}{*}{ Post-train } & Pre-exercise & $6727.82 \pm 2246.19$ & $51.95 \pm 16.79$ & $2.33 \pm 1.30$ \\
\hline & & Post-exercise & $7586.08 \pm 1167.91$ & $69.67 \pm 16.82^{\mathrm{a}}$ & $1.82 \pm 1.34$ \\
\hline \multicolumn{3}{|c|}{ Pre-test $P$-value (one-way ANOVA) } & 0.07 & 0.06 & 0.06 \\
\hline
\end{tabular}

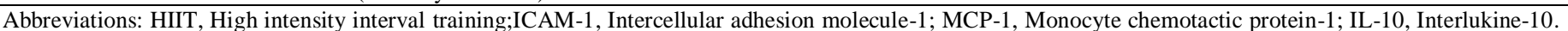

${ }^{a}$ Significant Difference Compared to Pre-exercise at $P<0.05$.

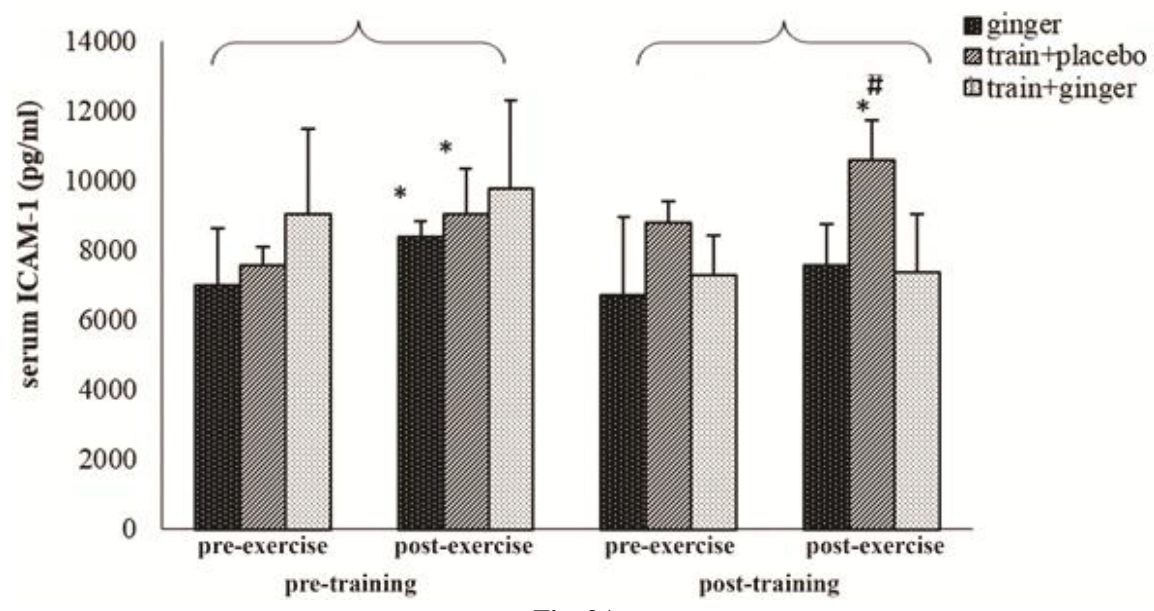

Fig. 3A:

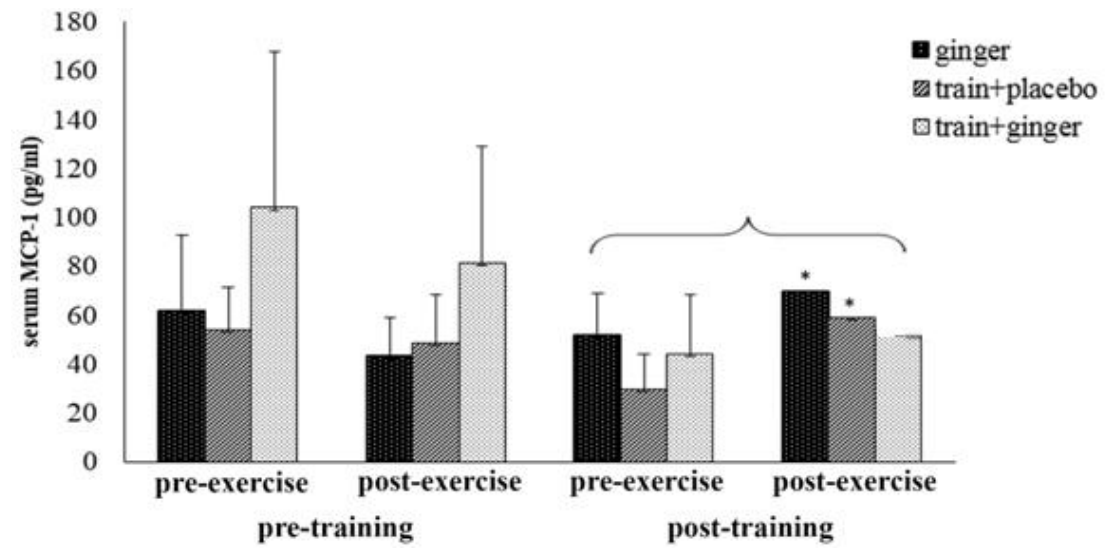

Fig. 3B:

Effects of training and ginger on the serum inflammatory markers in response to acute exercise

Two way repeated measure ANOVA and one way ANOVA test results are as follows:

As shown in table 3, serum ICAM-1 increased following the first $(P=0.04),(P=0.04)$ and second $(P=0.23),(P<0.001)$ acute exercise in ginger and HIIT + placebo groups respectively.
In addition, serum ICAM-1 insignificantly increased following the first $(P=0.48)$ and second $(P=0.23)$ acute exercise in HIIT + ginger group. The one way ANOVA showed no significant difference between three groups in serum ICAM-1 in response to the first acute exercise $(P=0.76)$ but showed significant increase in HIIT + placebo group in comparison with HIIT + ginger group in response to second acute exercise $(P=0.02)$ (Figure $3 \mathrm{~A})$. 


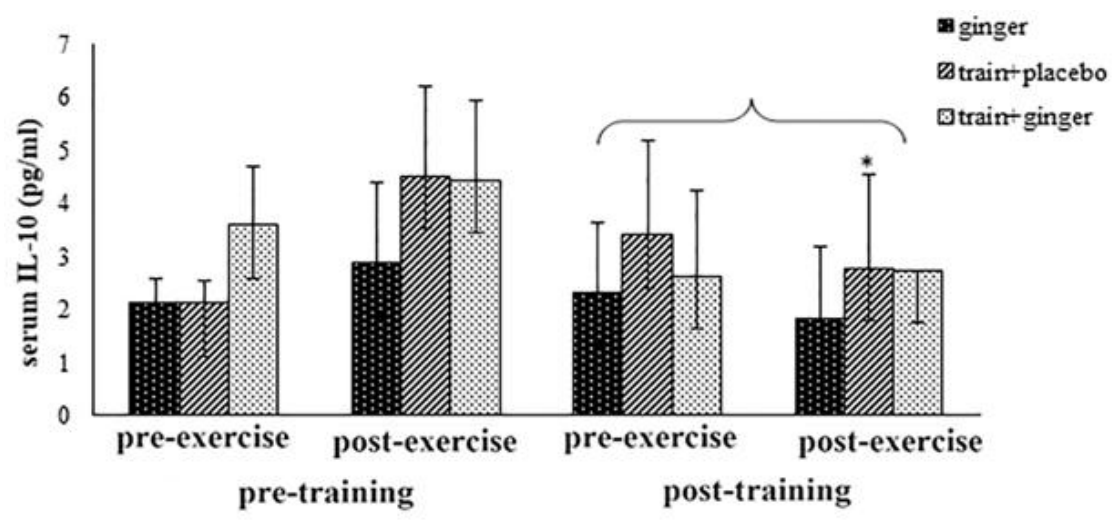

Fig. 3C:

Fig. 3: The effects of training and ginger on the serum (A) ICAM-1, (B) MCP-1,(C) IL-10 in response to an acute exercise session. $* P<0.05$ Difference from prepost exercise. $\# P<0.05$ difference between groups.

Moreover, serum MCP-1 showed no significant difference in response to the first $(P=0.13),(P=0.57)$ and significant increase in response to second $(P=0.01),(P=0.01)$ acute exercise in ginger and HIIT + placebo groups respectively. Also, there was no significant difference in serum MCP-1 in response to the first $(P=0.22)$ and second acute exercise in HIIT+ ginger group $(P=0.06)$. One way ANOVA showed no significant difference between three groups in serum MCP-1 in response to the first $(P=0.6)$ and second $(P=0.07)$ acute exercises. (Figure $3 \mathrm{~B})$

Serum IL-10 insignificantly increased in response to first $(P=0.21)$, and insignificant decrease in response to second $(P=0.32)$ acute exercise protocol in ginger group. In the other group, no significant change in serum IL-10 in response to the first $(P=0.7)$ and significant decrease in response to second acute exercise in HIIT + placebo group $(P=0.02)$ was shown. Also, insignificant increase in response to first $(P=0.2)$ and insignificant increase in response to second $(P=0.74)$ acute exercise in HIIT + ginger group $(P=0.74)$. One way ANOVA showed no significant difference between three groups in serum IL-10 in response to the first $(P=0.4)$ and second acute exercise $(P=0.32)$. (Figure 3C)

\section{DISCUSSION}

The aim of this study was 2 -fold. First, we sought to examine the effect of 10 weeks of short-term high-intensity interval training (40-m maximal Shuttle run) induced changes in serum ICAM-1, MCP-1 and IL-10 levels following an acute highintensity interval exercise challenge performed at the same absolute intensities as those undertaken prior to training. Second, since ginger is an anti-inflammatory plant, we aimed to determine if ginger consumption together with repeated acute exercise exposure (i.e., training) or alone, affects the serum inflammatory markers response to an acute exercise stress. The important findings of the study were that in overweight females, a $10 \mathrm{wk}$ HIIT regimen together with ginger could attenuate serum ICAM-1 more than two other groups in response to acute exercise, moreover an improved $\mathrm{PBF}$ and $\mathrm{Vo}_{2}$ max was also observed. No significant difference in serum MCP-1and IL-10 between groups were detected. The use of ICAM-1as a biomarker of endothelial dysfunction that can independently predict cardiovascular diseases (CVD) has been documented previously (Ridker et al., 1998). In the present study, we observed an insignificant reduction in serum ICAM-1in response to acute exercise after ginger and HIIT + ginger compared to HIIT + placebo. In other words, probably ginger affect to some extent in an anti-inflammatory way. It has been documented that 6-shogaol, the active element in ginger, decrease the ICAM-1gene expression transcription (Pan et al., 2008). Tonjes et al. (2007) in their study on 20 subjects, with natural glucose tolerance, after four weeks of severe aerobic exercises observed an insignificant change in their ICAM-1, although in another 20 individuals with glucose tolerance complication, a significant decrease was observed. Two other studies, also, have reported improvement of ICAM-1 in subjects with metabolic complication and type II diabetes due to aerobic exercises (Roberts et al., 2004, Zoppini et al., 2006). In all these studies pre-test ICAM-1 values were 2-3 times more than those of the present study. It is obvious that the subjects in these studies were suffering from different disorders; but the subjects in the present study were healthy. Very few studies have dealt with the combined effect of exercises and ginger consumption on inflammatory indices. Ayaz et al. (2012) has mentioned plasma decrease values of IL-6 and CRP as a result of physical exercises together with ginger consumption. Atashak et al. (2011) studied the effect of a ten-week progressive resistance exercises, accompanied by ginger consumption, on inflammatory indices in the obese men. Resistance exercises performing 50\%-75\% 1RM, by itself or together with ginger supplement led to a significant decrease in TC. But, the indices LDL, HDL, and TG did not significantly change. Black et al. (2011) and Mashhadi et al. (2013) too, did not find a combined effect of ginger and physical activity on inflammatory indices, like CRP and IL-6. On the other hand, present researchers couldn't observe the positive effects of training combined with ginger on MCP-1 and IL-10, while three groups affect similarly. This is in agreement with Connolley et al. (2004) who showed that the mRNA level of IL-10 was not affected after cycle ergometer workout at any time point. 
Zwetsloot et al. (2014) studied the effect of one severe activity session and a two-week HIIT on an ergometer (i.e. 6 HIIT sessions with 6-12 sixty _ second intervals and 75 seconds of active recovery) corresponding to $100 \% \mathrm{Vo}_{2} \max$ on 8 active men. Their serum samples were assessed after the first session and the end of the sixth. However, a two-week HIIT did not cause a change in the inflammatory response to one exercise session with respect to serum IL-10 and MCP-1. While, Reihmane et al. (2012) showed significant increase in MCP-1 and no change in ICAM-1 immediately after an acute sub maximal cycle exercise $(70 \%$ reserved heart rate, for $1 \mathrm{hr}$ ) in 7 elite and 10 amatory athletes. The mechanism interfere with causes change in ICAM-1 and MCP-1 after acute exercise might be due to increased pro inflammatory markers such as IL- 6 and TNF- $\alpha$ and shear stress during acute exercise that activates endothelium and monocytes, both of which may release MCP-1. The difference in participant's physical fitness in Reihmane et al. (2012) study and present paper may be the cause of discrepancy. In a study by Leggat et al. (2012), 2 weeks of high intensity interval training (10 intervals, $4 \mathrm{~min}$, 89.5\% maximum heart rate) decreased MCP-1significantly while no change was observed in serum IL-10 and ICAM-1in 12 obese and overweight men, and their $\mathrm{Vo}_{2} \max$ improved significantly. In both of aforementioned studies, participants were men whereas women took part in the present study. It has been said that in female participants the menstrual cycle itself affect the immune system. In the luteal phase, the concentration of leukocyte and lymphocyte subsets tend to be higher at rest and also after exercise, a statistically significant different pattern of gene regulation is found, with particularly an up regulation of proinflammatory genes compared to the follicular phase (Northoff $e t$ al., 2008). Since our participants were in their follicular phase, maybe the pro inflammatory subsets showed lesser, so the training and exercise couldn't influence effectively, and it can be a cause of discrepancy. Significant decrease in PBF after 10 weeks of HIIT + Ginger is the other finding of study. In addition, PBF significantly decreased in Ginger + HIIT rather than Ginger or HIIT + placebo, which means the combination of training and Ginger consumption result in maximum favorable results in PBF. This is in agreement with some studies (Tjonna et al., 2007). It is interesting that the training duration in afore mentioned investigations were longer $>10$ wks than present study, while this paper demonstrated the effects of HIIT in improvement of PBF in shorter period in overweight women. On the other hand, Astorino et al. (2013) and Keating et al. (2014) reported no change in PBF after 12 weeks of HIIT training in overweight men and women. Probably, the kind of trainings applied in these two papers (exercise on ergo meter) vs. present research (40-m shuttle-run) accounts for differences in body composition results. Moreover, in training groups, $\mathrm{Vo}_{2} \max$ improved almost equivocally, which emphasis on the effective role of training variable. This is in agreement with results of some studies (Astorino et al., 2013, Helgerud et al., 2007). It should be explained that increase in exercise capacity along with exercise intensity is related to near relationship between stroke volume and training intensity. Stroke volume augment parallel to exercise intensity, regarding maximum stroke volume is near to $\mathrm{Vo}_{2}$ max (Zwetsloot et al., 2014), so HIIT is a drastic method to improve $\mathrm{Vo}_{2}$ max. It has been documented that increase in $\mathrm{Vo}_{2} \max$ is related to decrease in percent body fat in young people (Sadhan $e t$ al., 2007). In the same way, improved $\mathrm{Vo}_{2} \mathrm{max}$ was accompanied by low percent body fat in Ginger + HIIT group, in present study. As a whole, we can suggest that an acute HIIT exercise session mimics the inflammatory status similar to inflammatory chronic disease in body by increasing inflammatory and anti-inflammatory markers, while such mentioned HIIT training for 10 weeks in combination with ginger intake can ameliorate to some extent the selective inflammatory markers. Probably if there was a control group we could conclude more precisely. A number of factors such as individual genetic variability, different exercise protocols, the heterogeneous nature of HIIT itself, and other lifestyle factors influence the effects observed in different papers. The training period was to some extent long, and it is likely to have changed parameters independent of training, and also concerning low number of samples, not including the control group in the present study, future studies should consider introducing a control group with more number of samples and if greater health benefits can be achieved over a longer training periods or if lower doses of ginger could be effective.

\section{CONCLUSION}

These findings demonstrate that high intensity interval training is a time efficient means for improving inflammatory status, physical fitness and body composition. Ginger has effective anti-inflammatory impacts on inflammatory markers especially in combination with training. Probably, athletes who take part in acute severe sports can benefit from HIIT to decrease their body inflammatory spectrum.

\section{ACKNOWLEDGEMENTS}

We appreciate the Dineh Iran Company for giving ginger and placebo tablets and Birjand University for supporting this study which is a part of a $\mathrm{PhD}$ thesis.

\section{REFERENCES}

Abd El- Kader SM, Gari AM, Salah El-Den AEM. Impact of moderate versus mild aerobic exercise training on inflammatory cytokines in obese type 2 diabetic patients: a randomized clinical trial. Afr Health Sci, 2013; 13(4): 857-863.

Atashak S, Peeri M, Azarbayjani MA, Stannard SR, MosalmanHaghighi M. Obesity-related cardiovascular risk factors after long term resistance training and ginger supplementation. J Sports Sci Med, 2011; 10(4):685-691.

Astorino TA, Schubert MM, Palumbo E, Stirling D, McMillan DW,Cooper C, et al. Magnitude and time course of changes in maximal oxygen uptake in response to distinct regimens of chronic interval training in sedentary women. Eur J Applphysiol, 2013; 113(9): 2361-2369.

Ayaz A, DabidiRoshan V. Effects of 6-weeks water based intermittent exercise with and without ZingiberOfficinale on proinflammatory markers and blood lipids in overweight women with breast cancer. J Appl Pharm Sci, 2012; 2(5):218-224. 
Bartlett DB, Shepherd SO, Wilson OJ, Taylor A, ThogersenNtoumani C, Wagenmakers AJM, et al. Can high intensity interval training reduce the risk of inflammatory and immune mediated mortality and morbidity with age? 64TH Annual scientific meeting, exercise, activity and ageing mechanisms; July ${ }^{7 \text { th }}-9^{\text {th }}$; Liverpool John Moores University, Liverpool, United Kingdom 2014.

Black CD, Herring MP, Hurely DJO, Connor PJ. Ginger reduces muscle pain caused by eccentric exercise. J Pain, 2011; 11(9): 894-903.

Buchan DS, Ollis S, Young JD, Thomas NE, Cooper SM, Tong $\mathrm{T}$, et al. The effects of time and intensity of exercise on novel and established markers of CVD in adolescent youth. Am J Hum Biol, 2011; 23(4):517-526.

Connolly PH. Effects of exercise on gene expression in human peripheral blood mononuclear cells. J Appl Physiol, 2004; 97(4):1461-69.

Dill DB, Costill DL. Calculation of percentage changes in volumes of blood, plasma and red cells in dehydration. J ApplPhysiol, 1974; 37(2):247-8.

Fatehi Z, Gholamnezhad Z, Jafarzadeh M, Fatehi M. The antiinflammatory effects of Aqueus extract of ginger root in diabetic mice. Daru, 2005; 13(2):70-73.

Helgerud J, Hoydal K, Wang E, Karlsen T, Berg P. Aerobic high intensity intervals improve Vo2max more than moderate training. Med Scie Sports Exerc, 2007; 39(4): 665-71.

Jackson AS, Pollock ML, Ward A. Generalized equations for predicting body density of women. Med Sci Sports Exerc, 1985; 12(3):175-75.

Keating SH, Machan EA, OConnor HT, Gerofi JA, Sainsbury A, Caterson ID, et al. Continuous exercise but not high intensity interval training imporoves fat distribution in overweight adults. J Obes, 2014; doi: $10.1155 / 2014 / 834865$

Leggat M, Carter WG, Evans MJC, Vennard RA, SribalaSundaram S, Nimmo MA. Determination of inflammatory and prominent proteomic changes in plasma and adipose tissue after high-intensity intermittent training in overweight and obese males. J ApplPhysiol, 2012; 112(8): 1353-1360.

Libby P. Inflammation in atherosclerosis. Nature, 2002; 420(6917):868-874

Little JP, Safdar A, Bishop D, Tarnopolsky MA, Gibala MJ. An acute bout of high - intensity interval training increases the nuclear abundance of PGC- $1 \alpha$ and activates mitochondrial biogenesis in human skeletal muscle. Am J PhysiolRegul, Integr Comp Physiol, 2011; 300(6):R1303-R1310.

Mashhadi NS, Ghiasvand R, Askari G, Hariri M, Darvishi L, Mofid MR. Anti-oxidative and anti-inflammatory effects of ginger in health and physical activity: review of current evidence. Int J Prev Med, 2013; 4(1):s36-42.

Nicoll R, Henein MY. Ginger (Zingiberofficinale Roscoe): A hot remedy for cardiovascular disease? Int J Cardiol, 2009; 131(3):408-9.

Nielsen HG, Lyberg T. Long-distance running modulates the expression of leucocyte and endothelial adhesion molecules. Scandinavian J Immun, 2004; 60(4):356-362.

Northoff H, Symons S, Zieker D, Schaible EV, Schafer K, Thoma S, et al. Gender and menstrual phase dependant regulation of inflammatory gene expression in response to aerobic exercise. Exercimmunol rev, 2008; 14:86-103.

Pan MH, Hsieh MC, Kuo JM, Lai CS, Wu H, Sang S. [6]Shogaol induces apoptosis in human colorectal carcinoma cells via ROS production, Caspase activation, and GADD 153 expression. MolNutr Food Res, 2008; 52(13):527-37.

Reihmane D, Tretjakovs P, Kaupe J, Sars M, Valante R, Jurka A. Systemic pro-inflammatory molecule response to acute submaximal exercise in moderately and highly trained athletes. Environ ExperiBiol, 2012; 10:107-112.

Ribeiro F, Alves AJ, Teixeira M, Miranda F, Azevedo C, Duarte JA, et al. Training increases interleukin-10 after an acute myocardial infarction: a randomised clinical trial. Int J Sports MedExerc, 2012; 33(3):192-8.

Ridker PM, Hennekens CH, Roitman-Johnson B, Stampfer MJ, Allen J. Plasma concentration of soluble intercellular adhesion molecule 1 and risks of future myocardial infarction in apparently healthy men. Lancet, 1998; 351(9096): 88-92.

Roberts CK, Won D, Pruthi S, Kurtovic S, Sindhu RK, Vaziri $\mathrm{ND}$, et al. Effect of a short-term diet and exercise intervention on oxidative stress, inflammation, MMP-9 and monocyte chemotactic activity in men with metabolic syndrome factors. J Applphysiol, 2006; 100:1657-1665.

Sadhan B, Koley SH, Sandhu S. Relationship between cardio respiratory fitness, body composition and blood pressure in Punjabi collegiate population. J Hum Ecol, 2007; 22(3):215-219.

Sallam N, Laher I. Exercise Modulates Oxidative Stress and Inflammation in Aging and Cardiovascular Diseases. Hindawi Publishing Corporation, Oxidative Medicine and Cellular Longevity, 2015; doi.org/10.1155/2016/7239639.

Sijie T, Hainai Y, Fengying Y, Jianxiong W. High intensity interval training in overweight young women. J Sports Med and Physical fitness, 2012; 52(3):255-62.

Simpson RJ, Florida-James GD, Whyte GP, Guy K. The effects of intensive, moderate and downhill treadmill running on human blood lymphocytes expressing the adhesion/activation molecules CD54 (ICAM1), CD18 (beta2 integrin) and CD53. Eur J ApplPhysiol, 2006; 97(1):10921.

Smith LL, McKune AJ, Semple SJ, Sibanda E, Steel H, Anderson R. Changes in serum cytokines after repeated bouts of downhill running. ApplPhysiolNutrMetab, 2007; 32(2): 233-240.

Talanian JL, Galloway SDR, Heigenhauser GJF, Bonen A, Spriet LL. Two weeks of high- intensity aerobic interval training increases the capacity for fat oxidation during exercise in women. J ApplPhysiol, 2007; 102(4):1439-1447.

Tjonna AE, Lee SJ, Rognmo O, Stolen TO, Bye A, Haram PM, et al. Aerobic Interval training versus continues moderate exercise as a treatment for the metabolic syndrome: a pilot study. Circulation, 2007; 118(4):346-54.

Tonjes A, Scholz M, Fasshauer M, Kratzsch J, Rassoul F, Stumvoll M, et al. Beneficial effects of a 4-week exercise program on plasma concentrations of adhesion molecules. Diabetes Care, 2007; 30(3):e1.

Verma SK, Singh M, Jain P, Bordia A. Protective effect of ginger, ZingiberofficinaleRosc on experimental atherosclerosis in rabbits. Indian J Exp Biol, 2004; 42(7):736-8.

Vrousgos G. Lifestyle Factors that Can Induce an Independent and Persistent Low-Grade Systemic Inflammatory Response: A Holistic Approach. The Open Biomarkers Journal, 2015; 7:1-28.

Wang YK, Hong YJ, Yao YH, Huang XM, Liu XB, Zhang CY, et al. 6-Shogaol Protects against Oxidized LDL-Induced Endothelial Injuries by Inhibiting Oxidized LDL-Evoked LOX-1 Signaling .EvidBased Complement Altern Med, 2013; http://dx.doi.org/10.1155/2013/503521.

Wilmore JH, Costill DL. Physiology of Sport and Exercise: 3rd ed. Champaign IL: Human Kinetics; 2005.

Zoppini G, Targher G, Zamboni C, Venturi C, Cacciatori C, Moghetti $\mathrm{P}$, et al. Effects of moderate-intensity exercise training on plasma biomarkers of inflammation and endothelial dysfunction in older patients with type 2 diabetes. Nutrmetab cardio vasc, 2006; 16:543-549.

Zwetsloot KA, John CS, Lawrence MM, Battista R, Shanely RA. High intensity interval training induces a modest systemic inflammatory response in active,young men.J Inflamm Res,2014;7:9-17.

\section{How to cite this article:}

Afzalpour MS, Nayebifar SH, Kazemi T, Abtahi-Eivary S-H, Mogharnasi M. Determination of Atherosclerosis markers changes after HIIT and ginger consumption in response to acute exercise in overweight women. J App Pharm Sci, 2016; 6 (07): 078-084. 